
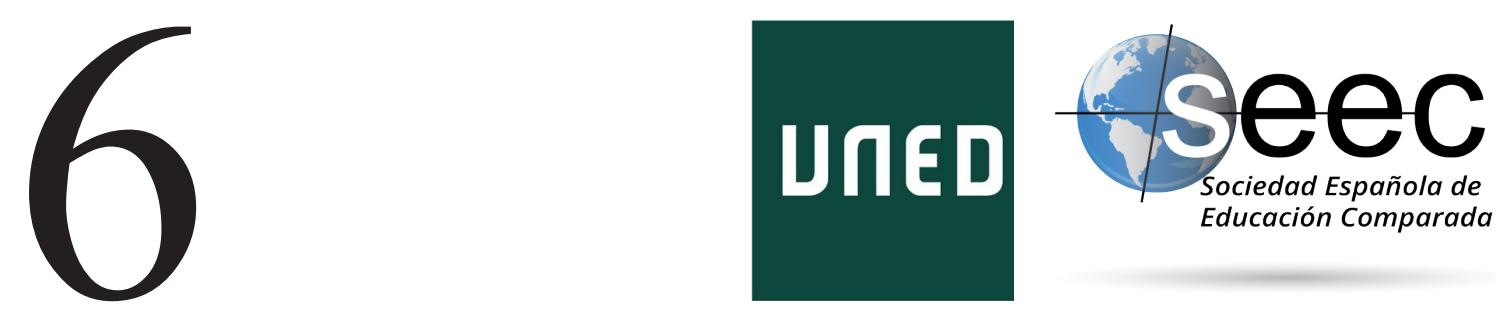

\title{
"Bildung": concepto, evolución e influjo en la pedagogía occidental desde una perspectiva histórica y actual
}

"Bildung": Concept, evolution and influence in Western
pedagogy from a historical and current perspective

\section{Alicia Sianes-Bautista*}

DoI: $10.5944 /$ reec.30.2017.18850

\section{Recibido: 25 de abril de 2017 Aceptado: 11 de julio de 2017}

\footnotetext{
* Alicia Sianes-Bautista: Pedagoga en Ábaco Centro de Psicología Infantil (Alcalá de Guadaíra, Sevilla). Profesora-Tutora de Historia de la Educación en el Centro Asociado de la UNED en Lanzarote durante el curso 2016/2017. Asistente Honoraria del Departamento de Teoría e Historia de la Educación y Pedagogía Social de la Universidad de Sevilla. Actualmente estudiante de Doctorado en Educación en la Universidad Nacional de Educación a Distancia, Departamento de Historia de la Educación y Educación Comparada. Líneas de investigación enfocadas a la Educación Comparada e Internacional, Educación Infantil, Aprendizaje a lo la vida, Pedagogía, Ciencias de la Educación, Educación Superior, EEES.. Datos de contacto: E-mail: sianes13@gmail.com
} 


\title{
Resumen
}

Alemania constituye un reconocido "país laboratorio» cuyo influjo clave en el pensamiento y la pedagogía contemporáneos ha sido reconocido unánimemente por los más reputados comparatistas académicos actuales. Especial influjo en la pedagogía occidental ha suscitado el concepto de Bildung, el cual, incardinado en el movimiento neohumanista alemán del siglo XVIII, es un concepto acuñado y desarrollado por Humboldt con ricas y múltiples acepciones, cuyo análisis se revela necesario a fin de desentrañar toda la riqueza de matices e implicaciones que su proyección es susceptible de verter en el ámbito educativo. Interesa para el corpus teórico de la disciplina pedagógica tener presente la relevancia educativa sin precedentes de este concepto alemán que en este artículo tomamos como objeto de estudio, y analizar su etimología y su significado, así como el influjo real de este término en la pedagogía occidental, no sólo desde un punto de vista histórico, sino analizando su impacto en el siglo XXI. A fin de llevar esto a cabo de forma eficaz, elegimos una metodología de carácter cualitativo, basada principalmente en el análisis de contenido mediante una minuciosa revisión de la literatura especializada sobre la temática objeto de estudio. En los resultados obtenidos quedan recogidos, entre otros, el origen etimológico de Bildung, además de su desarrollo conceptual a la vez que educativo a lo largo de la historia hasta llegar a confluir en el actual estado de la cuestión. En conclusión, se pone de manifiesto que dicho concepto ha sido tomado como un elemento singular precedente de multiplicidad de corrientes educativas y formativas no únicamente en la pedagogía occidental europea sino incluso también en la angloamericana, habiendo evolucionado hasta llegar incluso a concebirse como un elemento facilitador de los procesos educativos actuales, los cuales se encuentran inevitablemente enmarcados en la era de las TIC y la web 2.0.

Palabras clave: Bildung; Pedagogía; Occidente; Educación

\begin{abstract}
Germany is a recognized "laboratory country" which key influence on contemporary thinking and pedagogy has been unanimously recognized by the currently most respected academic comparatists. The Bildung concept has had a special influence on Western pedagogy, which, incardinated in the German 18th century's neohumanist movement, is a concept coined and developed by Humboldt with rich and multiple meanings, which analysis seems necessary for the purpose of unravelling all the wealth of nuances and implications that its projection is susceptible of generate in the education scope. It is interesting for the theoretical corpus of pedagogical discipline to bear in mind the unprecedented educational relevance of this German concept which has been taken as the object of study in this paper, and to analyse its etymology and meaning, as well as the real influence of this term in Western pedagogy, not only from a historical point of view, but also analysing its impact in the 21st century. In order to carry this out effectively, it has been applied a qualitative methodology, mainly based on the analysis of content through a detailed review of the specialized literature about the subject of study. The obtained results include, among others, the etymological origin of Bildung, in addition to its conceptual and educational development throughout history until it comes to converge in the current state of the issue. In conclusion, it is evident that this concept has been taken as a unique precedent of multiplicity of educational and formative currents not only in Western European but also in Anglo-American pedagogy, having evolved to the point of even conceiving itself as a facilitator of current educational processes, which are inevitably framed in the ICT and web 2.0 era.
\end{abstract}

Key Words: Bildung; Pedagogy; Western World; Education 


\section{Introducción}

Desde los tiempos del comparatista rumano Isaac L. Kandel, Alemania ha sido considerada de forma incuestionable como "país laboratorio» o modelo de primer orden en el terreno educacional (García Garrido, 2013). Resulta cierto que los académicos más experimentados intuyen que en el siglo XXI los países de referencia van a ser con probabilidad, otros. Pero no cabe ninguna duda de que los viejos modelos clásicos, como Alemania, aún tienen un gran peso en la conformación de la educación en el ámbito occidental (ídem: 21). Si bien el espíritu que tradicionalmente ha informado sus realizaciones educativas ha sido conceptualizado como dotado de rasgos de «conservadurismo antirreformista» (ídem: 33), resulta indiscutible el gran impacto e influjo alemán en la historia del pensamiento y de la pedagogía contemporáneas. En este sentido, uno de los conceptos pedagógicos de procedencia alemana más emblemáticos y más estudiados por académicos autóctonos y extranjeros, es el de Bildung, cuyo análisis ha ocupado una de las más extensas producciones bibliográficas del pensamiento pedagógico contemporáneo y actual. Acuñado y conceptualizado por Humboldt, el término y la realidad del vocablo Bildung se incardina en las corrientes de pensamiento pietista y neohumanista del siglo XVIII alemán, entendiéndose, en una de sus múltiples y más reconocidas acepciones, como un recorrido que el ser ha de perseguir hasta lograr su forma propia (Ipland García, 1999). La finalidad de la presente contribución apunta a desentrañar la etimología y los orígenes del concepto pedagógico germano de Bildung, así como a valorar el influjo real de este término en la Pedagogía occidental desde un enfoque tanto histórico como plenamente actual.

Desde un prisma metodológico, el presente estudio se lleva a cabo desde una perspectiva cualitativa, en concreto, a través de un análisis de contenido (Bardin, 2004) de los diversos documentos analizados.

\section{Etimología y concepto de Bildung}

$\mathrm{Si}$ atendemos en un primer momento al origen etimológico del concepto Bildung, nos encontramos ante un término que se caracteriza principalmente por traer implícita una amplia heterogeneidad y riqueza en cuanto a pensamientos y acepciones en que es susceptible de proyectarse en el ámbito educativo (Ipland García, 1999).

Así, cabe afirmar que desde un punto de vista etimológico, el concepto Bildung hunde sus raíces en la palabra Bild (imagen, cuadro,...). A partir de ella, derivó en el término bilden, que bien podría traducirse como «formar» hasta, finalmente, llegar a Bildung. Aun pudiendo emplear la traducción al español («formación») preferimos referirnos a él en este artículo empleando el término original alemán, al igual que hizo Ipland García (1999), para evitar posibles reduccionismos tanto terminológicos como nocionales. Así mismo, es preciso tener en cuenta que este término no hace únicamente alusión a un proceso que se ciña únicamente al marco intelectual o racional del ser humano, sino también a la parte más humana y dinámica del mismo. En esta misma línea Bildung, es un término que en el uso cotidiano en lengua alemana posee un significado bifurcado hacia procesos educativos generales y hacia los resultados obtenidos, se refiere en su sentido original a los procesos autoformativos (Fellenz, 2016).

Este concepto tiene, por tanto, una doble vertiente pues es posible entenderlo en un sentido activo y en otro pasivo. Su sentido activo haría referencia a Bildung como 
el proceso de formación, mientras que el pasivo implicaría además el resultado obtenido una vez finalizado dicho proceso (Quintana Cabanas, 1995). Así mostramos nuestro acuerdo con Menze (1981) cuando mantiene que:

«No existe definición con la cual se pueda establecer de un modo general el significado de Bildung. Sólo es posible decir que Bildung denota un proceso complejo por el cual debe producirse una personalidad estructurada según el modelo deseado» (p. 350)

Asimismo, es importante hacer mención a los conceptos alemanes que se encontraban estrechamente relacionados con la educación hasta la aparición de Bildung y que lo preceden: en primer lugar encontramos el concepto Erziehen, el cual es entendido como sinónimo de «educar», el cual posee acepciones de carácter religioso; Lernung, se concebía como «aprendizaje» y era un término que su empleo se ceñía a aquellos estudios de carácter científico; posteriormente, encontramos dos conceptos que se enfocan más hacia la formación que a la educación (formieren y bilden); Übung, se toma como equivalente a «ejercicio», aunque existía una importante diferenciación entre este y el de Unterricht («enseñanza»), al cual se le dotaba de un sentido más moral; finalmente, estaban los conceptos de erleuchten y aufklären, como sinónimos de aclarar.

Biesta (2016) clarifica las diferencias entre los conceptos de Bildung y Erziehung poniendo así de manifiesto que Bildung, es un término que hace alusión a la formación de uno mismo para con la cultura en la que inevitablemente se encuentra inmerso, digamos que podría concebirse como alcanzar la propia identidad. En este sentido, Bildung se asemejaría a la idea griega de Paideia (Jaeger, 1945; Nordenbo, 2002; Vergara Ciordia, 2014). Mientras que cuando no es uno mismo, sino la sociedad la que trata de ayudarnos a encontrar o establecer la identidad propia del individuo en relación con la cultura a la que pertenece, en alemán se haría uso del término Erziehung. A continuación presentamos una tabla en la que quedan recogidas las principales concepciones relativas a la dicotomía existente entre «educación» y «formación» o Erziehung y Bildung en la cultura neohumanista alemana por parte de los autores más representativos en la temática concerniente:

Tabla 1. Dicotomía entre Erziehung y Bildung. Fuente: Elaboración propia a partir de Ipland García (1999).

\begin{tabular}{|l|l|}
\hline Dilthey & $\begin{array}{l}\text { Erziehung es el punto de partida que configura el carácter de la persona. } \\
\text { Bildung posee sentido más amplio, de desarrollo personal, autosuperación } \\
\text { y comprenderse a uno mismo a la vez que al mundo. }\end{array}$ \\
\hline Willmann & $\begin{array}{l}\text { Dimensión intelectual de Bildung, capacidad para adquirir conocimiento; } \\
\text { la profundización en los temas, seleccionando lo más valioso; arraigo en lo } \\
\text { fundamental, discerniendo el valor de las cosas; comprensión del mundo y } \\
\text { del individuo mismo; alcanzar el término medio entre el diletantismo y la } \\
\text { labor profesional y técnica; la universalidad; las determinaciones morales y } \\
\text { la actitud ético-religiosa. }\end{array}$ \\
\hline Göttler & $\begin{array}{l}\text { Bildung presenta los valores al individuo a fin de que los comprenda y se in- } \\
\text { terese por ellos. Posteriormente la educación trataría que la persona sirviera } \\
\text { a los valores jerarquizados. }\end{array}$ \\
\hline Spranger & Bildung como categoría pedagógica superior, y capaz de producir cultura. \\
\hline
\end{tabular}


Digamos que Bildung (Becka, Solbrekkea, Sutphenb y Fremstada, 2015) se refiere a aquellos aspectos sutiles de la educación que van más allá, no ciñéndose taxativamente al conocimiento, recordándonos (Piercy, 2014) que existe también un camino más enfocado a la humanidad que al trabajo, y más cercano a la educación, que a las instituciones.

\section{Incidencia del neohumanismo en la conformación del término Bildung}

La comprensión de la completa profundidad del concepto de Bildung exige analizar mínimamente el movimiento filosófico en el que contextualmente emergió este término.

Resulta, pues, imperativo atender al neohumanismo alemán, movimiento que surgió como consecuencia de un momento albergador de una cadena de continuos cambios sociales que trajo consigo el cuestionamiento de los valores vigentes en la época. La corriente neohumanista busca entonces nuevos horizontes en los que no se persiga de forma taxativa la formación académica del individuo, sino que se le dote una notable importancia a la preparación para la vida en general (Ipland García, 1999). En esta línea, el neohumanismo termina por apoyarse en el entendimiento del ser humano como un ente global, como un todo, con la finalidad de lograr la consolidación de toda su estructura. De la mano de este movimiento llega la profundización del concepto de Bildung con las aportaciones de Humboldt y Herder, respectivamente. Los neohumanistas, entre los que se encuentran dichos autores, construyeron sobre muchas ideas enmarcadas en la filosofía idealista alemana, concibiendo Bildung como la cultivación de uno mismo y la libre interacción entre el individuo y los objetos culturales de la sociedad a la que pertenece (Hansen, 2008). Humboldt establece que no se pretende dejar más al descubierto el conocimiento del ser humano y, tomando únicamente como ayuda una sola parte de su formación (Bildung), conoce el objetivo que le ha sido fijado comprendiendo que es preciso dotar a su negocio de espíritu y una nueva visión del mundo obteniendo, por lo tanto, un estado de ánimo peculiar y nuevo de sí mismo, pudiendo él desde su punto de vista culminar su propia formación. Y esto es hacia dónde es preciso dirigirse (Humboldt, 1960). Asimismo, es menester que, junto con Bildung, la sabiduría y la virtud sean difundidas tanto como sea posible para que aumente su valor intrínseco tanto como para que el concepto de humanidad sea tomado como único ejemplo. Johann Gottfried von Herder, por su parte, pretendía desarrollar la idea de que Bildung no era sino el desarrollo de entenderse a uno mismo dentro de un contexto particular, social y cultural. La materialización de este entonces emergente concepto, basado en el neohumanismo y en la filosofía propia del idealismo alemán, ennoblece el carácter de Bildung (Hamann, 2011) no únicamente en las ciencias humanas, sino también en el desarrollo de la universidad alemana en todo su conjunto.

\section{Contenido y significaciones del concepto de Bildung}

Si nos dejamos guiar por la evolución de Bildung a lo largo de los siglos XIX y XX, terminamos por alcanzar el culmen de la pedagogía tradicional de la mano de Otto William. Él es quien empieza a hacer distinción entre «teoría de la educación», 
entendiéndola como equivalente a todo aquello que hoy albergamos bajo la denominación pedagogía y "teoría de la formación», concibiéndola como didáctica (Quintana Cabana, 1995). Bildung conforma un concepto crítico cuya responsabilidad radica en la propia persona y en la contribución hacia su formación íntegra y que toma como punto de partida la tradición de las artes liberales (Reindal, 2013). Asimismo, mostramos un gráfico en el que se observa la evolución histórica del uso de los términos Bildung, Erziehung y Aufklärung.

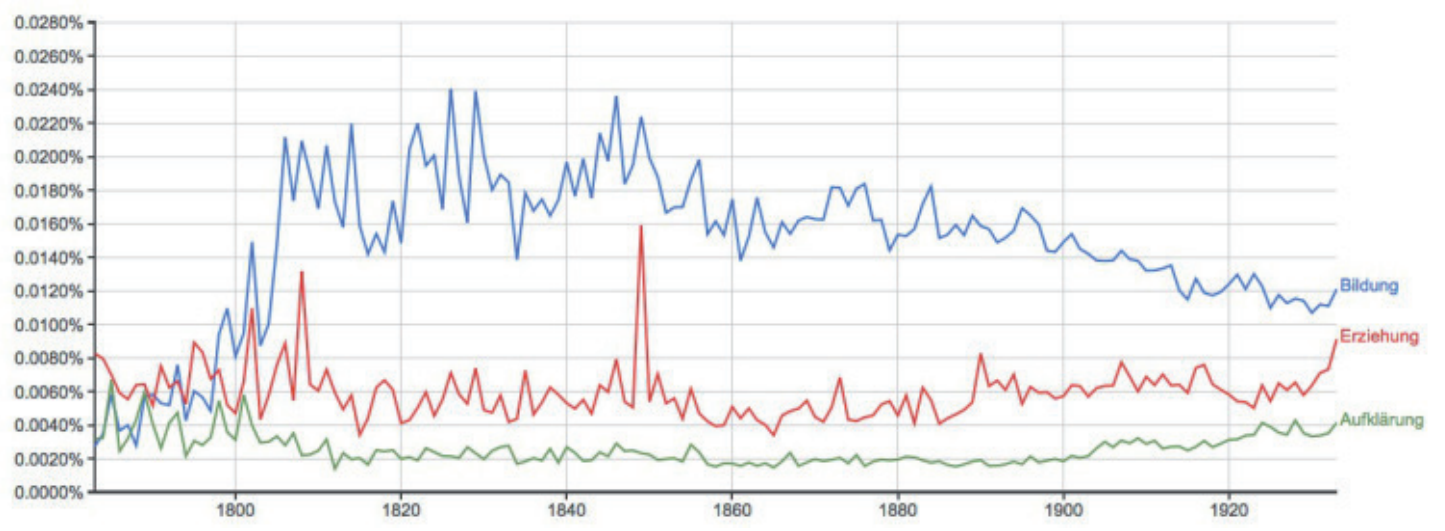

Gráfico 1: Trayectoria de Bildung, Erziehung y Aufklärung S. XIX. Fuente. Persistent Enlightment (2014).

Es posible distinguir como desde finales del siglo XVIII coexisten dichos términos, encontrándose además muy cercanos entre sí, a veces incluso empleados como sinónimos hasta el siglo XIX donde su distinción se hace cada vez más notoria: mientras que Aufklärunf se mantiene en unos niveles que oscilan entre el $0.0060 \%$ a inicios del siglo XIX y el 0.0040 \% en la segunda década del siglo XX; Erziehung experimenta altibajos que lo hacen posicionarse alrededor del $0.0130 \%$ a inicios del siglo XIX e incluso superior al 0.0140 \% a mediados del siglo XIX, no obstante, su influencia decae y se va manteniendo estable a niveles inferiores durante el resto de siglo y también entrado el siglo XX; finalmente, Bildung es el concepto que más se desarrolla en el siglo XIX, el cual se asociaba con la clase media y su pretensión de distinguirse de las masas no alfabetizadas (Hansen, 2008). En este momento alcanza niveles que rondan los $0.0240 \%$ durante la primera mitad de dicho siglo, posteriormente estos niveles van descendiendo progresivamente hasta la segunda década del siglo XX donde su porcentaje asciende únicamente al $0.0120 \%$.

Bildung engloba tres procesos sencillamente identificables (Deimann y Farrow, 2013; Hardy, Salo y Rönnerman, 2015; Becka, Solbrekkea, Sutphenb y Fremstada, 2015): en primer lugar sería posible distinguir los procesos de ser y el convertirse en un ser humano; en segundo lugar, se encuentra la interacción entre el individuo y la sociedad o el mundo; y, finalmente, las prácticas a partir de las cuales se organiza la influencia pedagógica a fin de potenciar la autoformación y ayudar al desarrollo del individuo racional. Kafki (1998) denomina estos procesos como autodeterminación (Self-determination), la determinación conjuntamente con los demás (Co-determination) y la solidaridad para con el resto y sus derechos (Solidarity) 
Por su parte autores como Humboldt y Hegel, entre otros, sugieren que Bildung está implicado a niveles muy profundos en la construcción de una estructura que media entre lo individual y el mundo (Franzel, 2014). Es decir, Bildung trae implícito el desarrollo de la cultura humana, dentro de la cual la investigación científica es una parte influyente (Pikkarainen, 2012).

\section{Impacto de Bildung en la pedagogía occidental}

Conviene no olvidar que Bildung, a pesar de haberse desarrollado en múltiples ámbitos a lo largo de la historia hasta llegar a convertirse en una de las nociones principales de la tradición educativa moderna en occidente (Biesta, 2002), es un concepto que está profundamente implicado en la cultura oficial alemana, en su vida académica e identidad nacional, además de conformar una operación que gestiona la parte y el todo en multiplicidad de formas a través de una variedad de contextos históricos (Franzel, 2014). De hecho muchas de las teorías relativas a Bildung en los niveles de educación secundaria y superior surgieron a partir de la reforma del currículum humanista mediante la filosofía idealista.

A finales del siglo XX, (Biesta, 2002) durante la década de los sesenta, el concepto de Bildung desapareció de la escena educativa europea. Esto fue así debido a que en muchos países europeos el discurso educativo sufrió un giro experimental en el que la noción primaria de Bildung fue sustituida por otras de carácter más psicológico y sociológico como la cualificación, la socialización, la integración y el aprendizaje. Asimismo, en los ochenta, emergió nuevamente el interés por Bildung, el cual se veía inevitablemente contextualizado por el debate acerca de la educación general ${ }^{1}$ (Horlacher, 2015), es decir, sobre el tipo de educación no vocacional con la que cada uno ha de comprometerse. No ha sido hasta tiempos recientes (Hansen, 2008) cuando se ha extendido y, por consiguiente, comenzado a emplear de forma más asidua el término Bildung también en la cultura anglo-americana.

Actualmente las preocupaciones principales del concepto de Bildung se encuentran focalizadas en la autoformación (Reichenbach, 2014), y la creación y transformación de relaciones específicas entre la formación, el propio individuo formado y el mundo (Bianchin, 2012; Fellenz, 2016). Asimismo Bildung ha sobrevivido también como temática en la teoría de la educación y la investigación (Hansen, 2008); y debido a sus múltiples desarrollos y carácter interdisciplinar a la vez que multidisciplinar, no es posible aportar una definición nominal de Bildung que aborde todos sus diferentes significados e implicaciones, de ahí la importancia de que sea problematizado y concretado para con diversas disciplinas y contextos (Reindal, 2013).

\section{Bildung y PISA}

En relación con el concepto alemán objeto de estudio en el contexto de PISA, resulta imprescindible hacer mención a un proceso fundamental que ha tenido lugar en Alemania con respecto a la reestructuración y a la redefinición de los objetivos educativos (Messner, 2016).

1 ‘allgemeine Bildung' o ‘Allgemeinbildung’ en alemán. 
Publicaciones recientes acerca de la temática que abordamos en el presente trabajo muestran que en la actualidad Bildung sigue siendo un término activo, en tanto que sigue relacionándose con nuevos conceptos y nociones, cada vez más enmarcadas en el propio contexto al que pertenecemos. Uno de los últimos conceptos con los que se ha vinculado el vocablo alemán es con el de competencia. Ya Roth había hecho previamente alusión al término de competencia (Horlacher, 2015), indicando tres posibles vertientes: la personal, la objetiva y la social, buscándose con ella la «disposición general de una persona para superar determinadas exigencias» (p. 90). No obstante, a partir del empleo de las competencias en estudios internacionales de rendimiento comparativo, entre los que destaca el Informe PISA de la OCDE, se toman las competencias como:

«sistema de destrezas, conocimientos y saberes metacognitivos y específicos y, en principio, susceptibles de aprendizaje, que permiten superar una clase de exigencias en entornos cotidianos, escolares y laborales» (Klieme, 2001, p. 182; Horlacher, 2015, p. 92).

No obstante, según Horlacher (2015) esto no hace más que generar debate y crítica pues, mientras que unos tratan de emplear el término 'competencia' como sinónimo de Bildung en algunos aspectos, otros mantienen que el primero únicamente se limita a las destrezas, a diferencia del segundo, que es ampliable a la toma de decisiones éticas o normativas, cuestiones aún pertinentes en la política educativa actual.

Las investigaciones de PISA en general, así como los argumentos basados en la literatura y en el concepto de competencia (Tenorth, 2016), son interpretadas como las piezas angulares de las cuestiones centrales relativas a educación básica (Grundbildung), es decir, que estas investigaciones no solo posibilitan la investigación educativa teórica sino que también aseguran la necesidad de dotar de empirismo a los programas teóricos clásicos.

La propia Gruschka (2015) manifiesta que no se puede prescindir del concepto de competencia, pues el discurso de las competencias es pedagógicamente razonable, siempre y cuando venga acompañada de la parte subjetiva de Bildung, palabra de la que procede. Así pues, mientras los debates acerca de las competencias siguen latentes, Klieme (2003) aprovecha para poner de manifiesto que, en este sentido, se considera que la educación no hace sino describir las habilidades de las que disponen los sujetos para ser capaces de actuar en condiciones de indecisión, incertidumbre, apertura y pluralismo, enmarcado en un proceso que posibilita la construcción personal del sujeto en vista a las inevitables expectativas sociales (Tenorth, 2016). No obstante, «no es posible enfatizar con la suficiente insistencia que los informes PISA no proyecte horizonte alguno para medir la educación general moderna ni tampoco describir la estructura de un currículum esencial» (Baumert et al. 2001, p. 21)².

\section{Bildung en el siglo XXI}

Actualmente nos hallamos en una sociedad caracterizada por encontrarse en constante devenir, en la que son frecuentes los cambios y además se producen a una velocidad vertiginosa. La educación, por tanto, se ve también inevitablemente afectada por ello. En

2 "Man kann gar nicht nachdrücklich genug betonen, dass PISA keineswegs beabsichtigt, den Horizont moderner Allgemeinbildung zu vermessen oder auch nur die Umrisse eines internationalen Kerncurriculums nachzuzeichnen”. 
el siglo XXI, uno de los cambios educativos más notorios se ha producido en la educación superior con la implantación del Plan Bolonia, en el cual se contemplan los tres ciclos de la educación superior: el Grado, el Máster y el Doctorado (Reindal, 2013). Se busca la armonía y estandarización de los sistemas de educación superior europeos (Fejes, 2008a, b), con la finalidad principal de desarrollar una Europa del Conocimiento (Declaración de Bolonia, 1999), puesto que está considerado un factor irreemplazable para alcanzar el crecimiento social y humano. No obstante, entendiendo el conocimiento como resultados de aprendizaje, la educación superior se convierte en un negocio de producción de conocimiento (Love, 2008).

La globalización no abarca únicamente a un hecho económico, sino también trae implícitas notables repercusiones que inciden en el carácter social, político y cultural de la sociedad. Lo cual implica que también la vida de las personas va cambiando y, con ella también su mentalidad y abren la mente hacia otras perspectivas educativas y formativas que previamente antes tal vez ni siquiera contemplaban (Hornstein, 2001). A día de hoy, Bildung se nos presenta todavía como un significativo objetivo educativo, incluso a pesar de no contribuir de forma directa a la eficiencia económica. Su potencial ha de basarse en la educación para la ciudadanía democrática y recordarle a los educadores los valores humanísticos propios de la modernidad como un elemento propio de una sociedad plural (Hansen, 2008). La globalización trae consigo también el surgimiento de nuevos objetivos formativos y la propagación de una nueva concepción de las personas. Asimismo, quien quiera cumplir con las exigencias y parámetros propios de la globalización debe ser también partidario de la flexibilidad, la movilidad, el espíritu de pertenecer a un equipo, etc. (Hornstein, 2001). De esta forma también se le otorga importancia al concepto de Bildung en relación con la integración de diferentes colectivos (adultos, personas con transfondos migratorios, etc. para con la sociedad actual del siglo XXI (Benner y Tenorth, 2000). Bildung debe entonces ser entendido (Deimann y Farrow, 2013) como una forma de reconsiderar las prácticas y los procesos de enseñanza-aprendizaje de una forma apropiada para afrontar adecuadamente los nuevos retos, no solo de la sociedad, sino también de la educación moderna. Bildung, se encuentra además estrechamente ligado al surgimiento de las universidades modernas (Franzel, 2014), pues existen planteamientos teóricos recientes en los que se pone de manifiesto que Bildung está más vinculada a las TIC que nunca (Deimann y Farrow, 2013). Es decir, se asume que los medios de comunicación son una herramienta esencial para Bildung, puesto que son las representativas de lo que es ahora el mundo (Jörissen y Marotzki, 2008) y, con su avance, Bildung queda efectivamente expuesto a nuevos retos, pero también a nuevas oportunidades. De hecho, si partimos de la base de que Bildung consiste, por un lado, en el desarrollo propio del individuo y, por otro, en la adecuada adaptación del mismo en el contexto y la sociedad en la que vive, fomentando una cultura participativa. No resulta, por tanto, en absoluto baladí pensar en vincular Bildung y las TIC, a fin de poder tomar también partida en la denominada era de la Web 2.0, puesto que actualmente son uno de los medios más influyentes en la sociedad occidental.

\section{Conclusiones}

A modo de colofón, es indudable que ya desde tiempos de la antigua Grecia con el surgimiento de la paideia, se le otorgaba una notable importancia al hecho de educar al individuo en todo su conjunto, de cultivarlo en cuerpo, mente y alma. Evitando, por tanto, 
ceñirse taxativamente al empleo de medidas instructivas y, tratando así de combinar este proceso con otras metodologías que ayudaran a enriquecer las diferentes facetas del ser humano. Es importante recordar, que las personas somos seres sociales, por lo que no basta únicamente con educar o formar al individuo para consigo mismo, sino que resulta imprescindible incluir adicionalmente valores sociales y culturales, a fin de que este pueda adaptarse de forma adecuada a la sociedad en la que se encuentra inevitablemente inmerso.

De ahí nace la importancia que le ha sido otorgada al término Bildung durante el proceso de conversión del individuo en persona y su adecuada inclusión social. Aludimos a un término tan complejo, heterogéneo y mutable que no solamente ha ejercido influencia sobre las corrientes educativas y pedagógicas en Alemania durante los siglos XVIII y XIX, sino que ha ido adaptándose a lo largo del tiempo a las nuevas demandas y requerimientos de la sociedad del momento. Ello hace que, en la sociedad actual que se encuentra en constante devenir y en la que el fenómeno de la Globalización resulta prácticamente incuestionable, Bildung no solo haya ejercido influencia en la pedagogía de Europa occidental sino que se haya extendido hasta llegar a ocupar un lugar incluso en las corrientes educativas anglo-americanas de la actualidad.

Asimismo, las Tecnologías de la Información y la Educación traen consigo multiplicidad de cambios a una velocidad vertiginosa, lo cual hace que las demandas sociales, educativas, culturales, etc. no hagan más que requerir una continua renovación y adaptación del individuo a la las nuevas exigencias. Por lo tanto, un hecho que podría traer de la mano la obsolescencia del término objeto de estudio, no hace sino incrementar su importancia aún hoy en el siglo XXI como herramienta clave para afrontar los retos educativos en cualquiera de sus niveles.

\section{Referencias bibliográficas}

AÑANOS-BEDRIÑANA, F. T. (2012): Pensamiento y acción socioeducativa en Europa y España. Evolución de la pedagogía y educación social, Revista Historia de la Educación Latinoamericana, 14, pp. 119-138.

BARDIN, L. (2004): Análise de conteúdo. (Lisboa, Edições 70).

BECKA, E. E., SOLBREKKEA, T. D., SUTPHENB, M. AND FREMSTADA, E. (2015): When mere knowledge is not enough: the potential of bildung as self-determination, codetermination and solidarity, Higher Education Research \& Development, 34, pp. 445-457.

BENNER, D. AND TENORTH, H. E. (2000). Bildungsprozesse und Lernen im Erwachsenenalter. Soziale Integration und Partizipation durch lebenslanges Lernen. Zeitschrift für Pädagogik, 42, pp. 69-90.

BIANCHIN, M. (2012): Bildung, meaning and reasons, Verifiche: Rivista Trimestrale di Scienze Umane 41, pp. 73-102.

BIESTA, G. (2002): How General Can Bildung Be? Reflections on the Future of a Modern Educational Ideal, Journal of Philosophy of Education, 36, pp. 377-390.

BIESTA, G. (2016): Who's Afraid of Teaching? Heidegger and the Question of Education ('Bildung'/'Erziehung'), Educational Philosophy and Theory, 48, pp. 832-845. 
CALZADILLA, R. (2004): La pedagogía como ciencia humanista: conocimiento de síntesis, complejidad y pluridisciplinariedad, Revista de Pedagogía, 25, pp. 123148.

PERSISTENT ENLIGHTMENT. (2014): Culture \& Civilization: The first English translation of Mendelssohn's answer to the question "What is Enlightenment?" Part II (https://persistentenlightenment.com/2014/04/20/mendelssohn2/), consultado el 30 de enero de 2017.

DEIMANN, M. AND FARROW, R. (2013): Rethinking OER and their Use: Open Education as Bildung, The International Review of Research in Open and Distributed Learning, 14, pp. 344-360.

FELLENZ, M. R. (2016): Forming the Professional Self: Bildung and the ontological perspective on professional education and development, Educational Philosophy and Theory, 48, pp. 267-283.

FEJES, A. (2008a): Standardising Europe: The Bologna Process and new modes of governing learning and teaching, Learning and Teaching The International Journal of Higher Education in the Social Sciences 1, pp. 25-49.

FEJES, A. (2008b): European citizens under construction: The Bologna Process analysed from a governmentality perspective, Educational Philosophy and Theory, 40, pp. 515-530.

GARCÍA GARRIDO, J. L. (1991): Fundamentos de la Educación Comparada. (Madrid, Dykinson).

GARCÍA GARRIDO, J. L. (2013): Sistemas educativos de hoy. ( $5^{\mathrm{a}}$ ed.) (Madrid, Ediciones Académicas).

GRIÉGER, P. (1972): Pedagogía general. (Alcoy, Editorial Marfil, S. A).

GRUSCHKA, A. (2015): Der Bildungs-Rat der Gesellschaft für Bildung und Wissen. Vorgelegt nach längerer Konsultation vom Präsidenten der Gesellschaft für Bildung und Wissen. (Opladen, Budrich).

FRANZEL, S. (2014): Recycling Bildung: From the Humboldt-Forum to Humboldt and Back, A Journal of Germanic Studies, 50, pp. 379-397.

HAMANN, J. (2011): 'Bildung' in German human sciences: the discursive transformation of a concept, History of the Human Sciences, 24, pp. 48-72.

HANSEN, K. H. (2008). Rewriting Bildung for Postmodernity: Books on Educational Philosophy, Classroom Practice, and Reflective Teaching. [Essay Review de los libros Educating Humanity: Bildung in Postmodernity, de L. Løvlie, K. P. Mortensen and S. E. Nordenbo; Looking Into Classrooms. Papers on Didactics, de P. Menck; and Teaching as a Reflective Practice. The German Didaktik Tradition, de I. Westbury, S. Hopmann and K. Riquarts].Curriculum Inquiry, 38, pp. 93-115.

HARDY, I., SALO, P., and RÖNNERMAN, K. (2015): Bildung and educational action research: resources for hope in neoliberal times, Action Research, 23, pp. 383-398. 
HORLACHER, R. (2015): Bildung, la formación. (Barcelona, Octaedro).

HORNSTEIN, W. (2001): Erziehung und Bildung im Zeitalter der Globalisierung. Themen und Fragestellungen erziehungswissenschaftlicher Reflexion. Zeitschrift für Pädagogik, 47, pp. 517-537

HOUSSAYE, J. (2014): Formar en pedagogía. Sí, ¿̇pero cómo? Estudios Pedagógicos, XL, pp. 275-283.

IPLAND GARCÍA, J. (1999): El concepto de Bildung en el Neohumanismo alemán. (Huelva, Hergue Editora Andaluza).

JAEGER, W. (1945): Paideia: The ideals of Greek culture. (New York, Oxford University Press).

JÖRISSEN, B., and MAROTZKI, W. (2008): Neue Bildungskulturen im Web 2.0: Artikulation, Partizipation, Syndikation, en F. VON GROSS, W. MAROTZKI and U. SANDER (Eds.) Internet Bildung Gemeinschaft, pp. 203-225. (Wiesbaden, Verlag für Sozialwissenschaften).

KLAFKI, W. (1998): Characteristics of critical-constructive Didaktik, en B.B. GUNDEM and S. HOPMANN (Eds.), Didaktik and/or curriculum. An international dialogue, pp. 307-330. (New York, Peter Lang).

KLIEME, E., AVENARIUS, H., BLUM, W., DÖBRICH, P. GRUBER, H., PRENZEL, M., REISS, K., RIQUARTS, K., ROST, J., TENORTH, H. and VOLLMER, H. J. (2003): Zur Entwicklung nationaler Bildungsstandards. Eine Expertise. (Berlin DIPF/ Bonn, BMBF).

LOVE, K. (2008): Higher education, pedagogy and the 'customerisation' of teaching and learning, Journal of Philosophy of Education, 42, p. 15-34.

MALDONADO, C. E. (2014): ¿Qué es eso de pedagogía y educación en complejidad?, Intersticios sociales, 7 , pp. 1-23.

MENDOZA, C. (2004): La pedagogía como ciencia: notas para un debate, Educación y postgrado, 9, pp. 229-240.

MENZE, C. (1981): Formación, en SPECK, J., WEHLE, G. Conceptos fundamentales en pedagogía. (Barcelona, Herder).

MESSNER, R. (2016): Bildungsforschung und Bildungstheorie nach PISA - ein schwieriges Verhältnis, Zeitschrift für Erziehungswissenschaft, 19, pp. 23-44.

NASSIF, R. (1983): Pedagogía general. (Madrid, Cincel-Kapelusz).

OLMOS DE MONTAÑEZ, O. (2009): Algunas ideas para la reconceptualización de la pedagogía como fundamento de la formación docente, (Paradigma, XXIX, pp. 7-29).

PIERCY, G. (2014): Becoming oneself: dimensions of Bildung' and the facilitation of personality development, Studies in Continuing Education, 36, pp. 380-382. 
PIKKARAINEN, E. (2012): Signs of Reality - The idea of General Bildung by J. A. Comenius, en P. SILJANDER, A. KIVELÄ and A. SUTINEN (Eds.), Theories of Bildung and Growth. Connections and Controversies between Continental Educational Thinking and American Pragmatism, pp.19-29. (Rotterdam, Sense Publishers).

QUINTANA CABANAS, J. M. (1995): El concepto de formación (Bildung) en el pensamiento alemán, en QUINTANA CABANAS, J. M. Teoría de la Educación. Concepción antinómica de la educación, pp. 33-45. (Madrid, Dykinson).

REICHENBACH, R. (2014): Humanistic Bildung: regulative idea or empty concept?, Asia Pacific Education Review, 15, pp. 65-70.

REINDAL, S. M. (2013): Bildung, the Bologna Process and Kierkegaard's Concept of Subjective Thinking, Studies in philosophy and education, 32, pp. 533-549.

SANVISENS, A. (1984): Introducción a la pedagogía. (Barcelona, Barcanova).

SENENT SÁNCHEZ, J. M. (2005): Los estudios de pedagogía en Europa en el contexto de la implantación del proceso de Bolonia y la situación de la Educación Comparada, Revista Española de Educación Comparada, 11, pp. 95-133.

TENORTH, H. E. (2016). Philosophy of Bildung and research on human development, Bildung and "basic education for all". An attempt to clarify relations, with a special respect to PISA. Zeitschrift für Erziehungswisenschaft, 19, pp. 45-71.

VERGARA CIORDIA, J. (2014): La educación en la antigua Grecia, en O. NEGRÍN FAJARDO and J. VERGARA CIORDIA, Historia de la Educación: de la Grecia clásica a la educación contemporánea. (Madrid: Dyckinson).

VON HILDEBRAND, D. and BARRIO MAESTRE, J. M. (2006): ¿Existe alguna legalidad o autonomía propia de la pedagogía?, Educación y Educadores, 9, pp. 87-101.

VON HUMBOLDT, W. (1960): Theorie der Bildung des Menschens, en W.VON HUMBOLDT, Schriften zur Anthropologie und Geschichte. (Stutgart, J. G. Cotta'sche Buchhandlung Nachfolger $\mathrm{GmbH}$ ). 